Rodríguez-Villalobos, J.C., \& Ayala-Bocos, A. (2021).

Depredación masiva por Acanthaster planci en el

arrecife El Corralito, golfo de California: amenaza

a corto plazo. Revista de Biología Tropical, 69(S1),

272-286. DOI 10.15517/rbt.v69iSuppl.1.46359

DOI 10.15517/rbt.v69iSuppl.1.46359

\title{
Depredación masiva por Acanthaster planci en el arrecife El Corralito, golfo de California: amenaza a corto plazo
}

Jenny Carolina Rodríguez-Villalobos ${ }^{1,2 *}$

Arturo Ayala-Bocos ${ }^{2}$

1. Universidad Autónoma de Baja California Sur. Departamento Académico de Ciencias Marinas y Costeras. Carretera al sur km 5.5, Col. El Mezquitito. CP 23080. La Paz, Baja California Sur, México; jcrv@ecoycon.org (*Correspondencia).

2. Ecosistemas y Conservación (Proazul Terrestre A.C). Calle Héroes de Independencia 2440. CP 23000. La Paz, Baja California Sur, México; arturobocos@ecoycon.org

Recibido 01-VII-2020. Corregido 27-VIII-2020. Aceptado 10-XII-2020.

\author{
ABSTRACT \\ Massive predation by Acanthaster planci in El Corralito, gulf of California: a short-term threat
}

\begin{abstract}
Introduction: One of the current threats to coral reefs is the loss of live coral cover. Massive predation associated with population outbreaks of the crown of thorns seastar, threatens the permanence of the reefs. Since 2017, there has been evidence of an increase in the density of the asteroid and an increase in coral mortality in the southern gulf of California. Objective: To describe the first event of massive predation on corals by Acanthaster planci in Espiritu Santo Island, gulf of California, Mexico. Methods: We visited El Corralito reef nine times between 2017 and 2019, using errant and band transect (25 x 3 m) visual censuses to determine Star density, behavior and damage. Over seven months, we monitored predation on colonies of Pavona gigantea. Results: The mean density of individuals in El Corralito in 2018-2019 was $607.40 \mathrm{ind} / \mathrm{ha}$. Asteroid predation was evident in more than $60 \%$ of diagnosed individuals $(\mathrm{N}=827)$, with the greatest affectations in the $P$. gigantea colonies ( $80 \%$ ). $63 \%$ of the 129 observed asteroid individuals were feeding mainly on Porites panamensis ( $68 \%$ of cases). Acute injuries indicate that the predation event is active. In 81 days, $25 \mathrm{~m}^{2}$ of one of the monitored colonies were lost. Conclusions: There is an active outbreak event with significant negative consequences on the El Corralito reef, which could determine the loss of coral cover in a few months. Monitoring and management are required to establish the reasons that led to the outbreak and to control it.
\end{abstract}

Key words: mortality; corallivory; reef degradation; outbreak; lesion.

Los arrecifes de coral enfrentan diversas amenazas que comprometen su permanencia a lo largo de su distribución geográfica (Birkeland, 2019). A escala global, las condiciones asociadas con el cambio climático (e.g. incremento de temperatura y acidificación del océano) que afectan a la comunidad de especies arrecifales (Hoey et al., 2016) y el incremento de enfermedades sobre las colonias coralinas (Peters, 2015) alteran la estructura y la función de los arrecifes de coral (Hoey et al., 2016). En combinación con las condiciones de cambio climático, localmente los arrecifes son afectados por la contaminación del agua que incrementa la vulnerabilidad de las especies ante otras amenazas tales como las enfermedades (França et al., 2020); así como la sobrepesca, la cual altera el equilibrio de las comunidades y la interacción entre las especies arrecifales, como la coralivoría (Rice, Ezzat, \& Burkepile, 2019). 
Los organismos coralívoros pertenecen a diferentes grupos marinos que incluyen anélidos, crustáceos, moluscos, equinoideos y peces, entre otros (Rotjan \& Lewis, 2008; Rice et al., 2019) y cumplen una función importante en los ecosistemas arrecifales puesto que su depredación selectiva equilibra la relaciones entre los corales y sus competidores (Rice et al., 2019) y favorece la reproducción asexual de los corales (Enochs \& Glynn, 2017). Sin embargo, cuando la depredación afecta negativamente a las colonias coralinas se disminuyen sus tasas de crecimiento y reproducción (Rotjan \& Lewis, 2008), y cuando ocurre de manera masiva, supera la velocidad de crecimiento de los corales y altera la dinámica de la comunidad por el incremento en las tasas de mortalidad de los individuos (Henry \& Hart, 2005). Esta muerte coralina causa alteraciones ecológicas (De'ath, Fabricius, Sweatman, \& Puotinen, 2012) asociadas con el cambio en la estructura del arrecife (e.g. disminución de la tridimensionalidad) y la comunidad de especies dependientes del coral para su protección (Pratchett et al., 2017). Asimismo, bajo ciertas circunstancias (e.g. disminución de herbívoros en el arrecife), la mortalidad producida por la depredación generalizada en el arrecife puede conducir incluso, al cambio de fase en los arrecifes llevándolos a ser dominados por especies altamente competitivas como las algas (Uthicke, Schaffelke, \& Byrne, 2009).

Uno de los depredadores coralinos más reconocidos es la estrella de mar corona de espinas Acanthaster spp., -excepto Acanthaster brevispinus-, (Haszprunar \& Spies 2014), un equinodermo omnívoro que se alimenta de tunicados, esponjas, algas y corales, y que ha recibido una gran atención en estudios arrecifales (Pratchett et al., 2017) debido a que es una de las principales amenazas para este ecosistema, principalmente en el océano IndoPacífico (Goldberg \& Wilkinson, 2004; Bruno \& Selig, 2007; De'ath et al., 2012). Existen diferencias en la morfología y en los hábitos alimenticios entre los individuos de Acanthaster spp. distribuidos en diferentes regiones geográficas, sin embargo, dentro del género solamente Acanthaster planci y A. brevispinus son las especies aceptadas como válidas en la actualidad (Horton et al., 2020). Estudios moleculares de ADN mitocondrial, han determinado que $A$. planci en realidad puede tratarse de un complejo de especies representados por cuatro clados claramente diferenciados (Vogler, Benzie, Lassios, Barber, \& Wörheide, 2008). De acuerdo con los hallazgos, los especímenes distribuidos en el Pacífico Oriental Tropical (POT) presentan semejanzas genéticas con la especie característica de los arrecifes del Pacífico, Acanthaster solaris (Haszprunar, Vogler, \& Wörheide, 2017), sin embargo, en este trabajo nos referiremos como A. planci a los individuos presentes en el golfo de California.

Las preferencias alimenticias de Acanthaster spp. sobre las especies de coral se relacionan con diversos factores tales como contenido nutricional y defensas del coral, forma de crecimiento colonial, presencia de especies comensales, condiciones ambientales locales y experiencias alimenticias previas del asteroideo (Birkeland \& Lucas, 1990; Keesing, 1990). Los componentes coralinos de la dieta de estas especies son diversos y sus preferencias son variadas: en la Gran Barrera Arrecifal se alimentan de corales de los géneros Acropora y Montipora y evitan aquellos del género Porites (De'ath \& Moran, 1988), y en el POT también se ha reportado alimentándose de corales del género Pocillopora (Glynn, 1976), Pavona, Gardineroseris, Porites, Psammocora, y Millepora (Maté, 2003). Diversos eventos de depredación masiva de corales (con evidencia de un daño general en el arrecife) se han detectado en arrecifes de la Gran Barrera Arrecifal (Pratchett et al., 2017), la Polinesia Francesa (Kayal et al., 2012) y el Mar Rojo (Moore, 1990), donde la densidad de los asteroideos incrementa por encima de $15 \mathrm{ind} / \mathrm{ha}$ (Moran \& De'ath, 1992) durante eventos conocidos como brotes poblacionales (o outbreaks), los cuales causan efectos negativos sobre las comunidades coralinas (De'ath et al., 2012). La definición de estos umbrales de crecimiento poblacional es un tanto elusivo debido a la ambigüedad en el establecimiento de las densidades normales 
de las poblaciones (Pratchett, Caballes, RiveraPosada, \& Sweatman, 2014) y a menudo son asignados a razón del nivel en el que la densidad del asteroideo genera mortalidad coralina importante en el arrecife (Moran \& De'ath, 1992), hasta el punto de superar el crecimiento de las colonias. En el caso del POT, Glynn (1973) estimó que densidades superiores a 260 ind/ha podrían causar rápidas destrucciones de los arrecifes en Panamá, considerando el crecimiento de las colonias coralinas y la depredación por los asteroideos.

Las poblaciones de invertebrados, en particular de aquellas especies con alta fecundidad como Acanthaster spp., presentan fluctuaciones importantes respecto al número de individuos (Uthicke et al., 2009), sin embargo, las causas por las cuales los brotes son más frecuentes e intensos aún no tienen una explicación generalizada (Pratchett et al., 2017). Pese a lo anterior, diversos factores naturales (e.g. dinámica propia de sus poblaciones) y antropogénicos (e.g. enriquecimiento de agua con nutrientes de uso humano, sobrepesca de depredadores del asteroideo) a escala local han sido postulados como probables causantes de los incrementos repentinos de individuos (Randall, 1972; Endean, 1974; Pratchett et al., 2014). De la misma manera, aún no es clara la razón por la cual, tras el crecimiento exponencial de la población y el consumo masivo de corales, se presentan disminuciones considerables en el número de asteroideos, aunque se piensa que se puede deber a la inanición de ellos por limitación en las presas (Cheser, 1969) y/o a su movimiento a otros arrecifes con disponibilidad de alimento (Endean, 1969), sin embargo el desplazamiento entre arrecifes es algo que aún no está estudiado (Pratchett et al., 2017).

El rápido crecimiento poblacional de Acanthasther spp. asociado con mortalidades altas de corales se había registrado en el Pacífico central, Indo-Pacífico, Australia, Mar Rojo y el sur de Japón (Branham, Reed, \& Bailey, 1971; Moran, 1986; Moore, 1990; Birkeland \& Lucas, 1990). Sin embargo, recientemente se registró un evento de mortalidad coralina asociado a la depredación de $A$. planci en arrecifes del Parque Nacional Zona Marina Archipiélago Espíritu Santo (golfo de California, México) donde la densidad estimada alcanzó 1500 ind/ha (Rodríguez-Villalobos \& Ayala-Bocos, 2018). En el golfo de California, A. planci se distribuye desde Cabo Pulmo $\left(23^{\circ} \mathrm{N}\right)$ hasta isla Rasa $\left(28^{\circ} \mathrm{N}\right)$, y se había reportado hasta 2017 con densidades máximas de 220 ind/ha (Herrero-Pérezrul, 2008) con prevalencias de lesión menores al $1 \%$ dentro de las comunidades (Rodríguez-Villalobos, Work, CalderonAguilera, Reyes-Bonilla, \& Hernández, 2015).

En consideración del potencial daño ecológico que el crecimiento excesivo de las poblaciones de Acanthasther spp. puede causar dentro de las comunidades coralinas (De'ath et al., 2012; Pratchett et al., 2017), y que por ende podría causar sobre los arrecifes del POT, el objetivo de este estudio es describir en el tiempo el evento de depredación masiva iniciado en 2017 por este asteroideo sobre los corales del El Corralito dentro del Parque Nacional Zona Marina Archipiélago Espíritu Santo, así como evaluar las consecuencias de este evento para el arrecife.

\section{MATERIALES Y MÉTODOS}

El arrecife El Corralito se encuentra localizado dentro del Parque Nacional Zona Marina Archipiélago Espíritu Santo (PNZMAES), Baja California Sur, México, en las coorde-

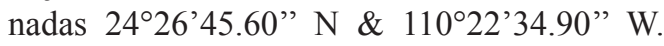
Este es un desarrollo arrecifal a manera de parches distribuidos discontinuamente en el oeste de la isla el cual es favorecido por una topografía que genera bahías protegidas aptas para el crecimiento coralino (Reyes-Bonilla \& López-Pérez, 2009). El Corralito es un arrecife de aproximadamente 1.3 ha, dominado principalmente por corales del género Pocillopora spp., y con presencia de las especies Porites panamensis y Pavona gigantea en menor abundancia. En el arrecife, la cobertura promedio de coral vivo es del $20 \%$. La profundidad máxima es de $6 \mathrm{~m}$ y la temperatura del agua oscila entre los $19^{\circ} \mathrm{C}$ en invierno y $29^{\circ} \mathrm{C}$ en verano (CONANP, 2014). 
Entre los años 2017 y 2019 se realizaron nueve visitas a El Corralito: con equipo de buceo SCUBA se realizó un buceo errante en julio 2017 y dos en agosto 2017 (cada uno de 45 minutos de duración). Además, se realizaron seis visitas más entre 2018 y 2019 en dónde se realizaron en total 18 transectos de banda de 25 $\times 3 \mathrm{~m}$ (seis en agosto 2018, cuatro en septiembre 2018, uno en mayo 2019, cuatro en agosto 2019 y tres en septiembre 2019) dispuestos de manera paralela a la línea de costa. A lo largo de los transectos se contabilizaron los individuos de $A$. planci para estimar la densidad de la población (ind/ha). Asimismo, se caracterizó el sustrato sobre el que se encontraba cada individuo (coral vivo, coral muerto, roca, bloque, alga calcárea, pedacería y otros) y se determinó si se encontraba alimentándose (de acuerdo con la postura globosa sobre el alimento y mediante la confirmación de la eversión de su estómago).

La prevalencia de lesiones asociadas con la depredación de $A$. planci fue estimada como el porcentaje de colonias de $P$. gigantea, $P$. panamensis y Pocillopora spp. afectadas por la alimentación del asteroideo dentro de los transectos realizados en 2018 y 2019. El diagnóstico de las colonias coralinas con una lesión asociada a la depredación por A. planci, se basó en las características de la huella que deja el asteroideo en las colonias, evidenciadas por pérdida de tejido focal o multifocal sin daño de estructuras esqueléticas. En el caso de las especies con crecimiento masivo ( $P$. gigantea y $P$. panamensis), las lesiones se presentan con morfología circular y de tamaño proporcional al disco de las estrellas (Fig. 1A). En el caso de las especies ramificadas (Pocillopora spp; Fig. 1B), la herida causada por la depredación se presenta como tejido expuesto sin daño esqueletal desde la punta hacia media altura de las ramas (alcance máximo del estómago en eversión de las colonias; Rodríguez-Villalobos et al., 2015). La prevalencia de depredación se evaluó a nivel poblacional para las especies de $P$. gigantea y P. panamensis y a nivel comunitario en el caso de Pocillopora spp. y considerando todas las especies evaluadas en el sitio. Las diferencias en la prevalencia entre los años de muestreo se evaluaron mediante la prueba de Mann-Whitney implementada con el software Statistica v7.1 (Statsoft Inc., 2005).

Como parte de la caracterización del evento de depredación sobre los corales en El Corralito, las lesiones infringidas por el asteroideo fueron categorizadas como agudas o subagudas, lo cual sugiere el tiempo de evolución de la herida (Work \& Aeby, 2006), por lo que aquellas lesiones con esqueleto expuesto de

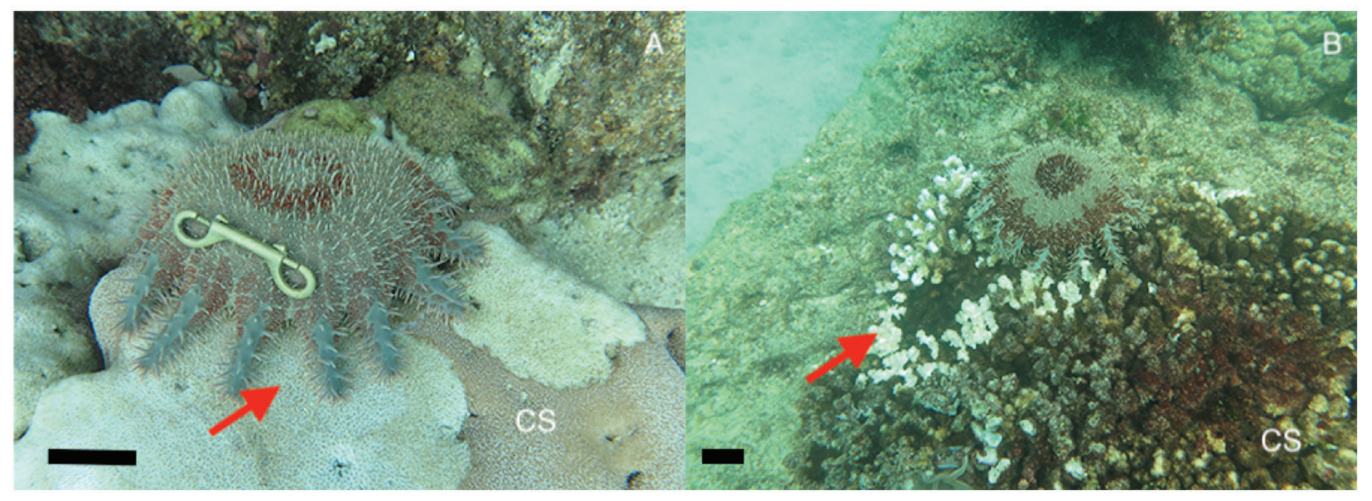

Fig. 1. Lesiones morfológicas causadas por la depredación de Acanthaster planci en A. Colonias de crecimiento masivo como Pavona gigantea, y B. Colonias de crecimiento ramificado como Pocillopora spp. Flechas rojas indican el esqueleto expuesto tras la depredación. Escala: $5 \mathrm{~cm}$. CS: coral sano.

Fig. 1. Morphological injuries caused by the predation of Acanthaster planci in A. Mass-growing colonies such as Pavona gigantea, and B. Branch-growing colonies such as Pocillopora spp. Red arrows indicate the exposed skeleton after predation. Scale: $5 \mathrm{~cm}$. CS: healthy coral. 
color blanco y sin sobre-crecimiento algal, se definieron como agudas e indican un tiempo de evolución de horas a días, y aquellas caracterizadas por esqueletos con sobre-crecimiento de algas se definieron como subagudas, e indican un tiempo de evolución de semanas.

Por último, en consideración al gran tamaño y el crecimiento lento de las colonias masivas, así como a la alta depredación ocurrida sobre $P$. gigantea en el área (Rodríguez-Villalobos \& Ayala-Bocos, 2018), se realizó el seguimiento fotográfico de la depredación entre el 18 de septiembre de 2018 y el 13 de mayo de 2019, sobre ocho colonias, hasta su mortalidad total. Se estimó la tasa de depredación como el área total depredada (área de la colonia estimada como una semi-elipse) entre el número de días transcurridos entre el estado saludable y la depredación completa de una de las colonias.

\section{RESULTADOS}

Durante los buceos errantes realizados en El Corralito en el año 2017, se contabilizó un total de 47 individuos de $A$. planci con un promedio de 15.6 individuos observados cada 45 minutos de buceo. Por otro lado, entre los años 2018 y 2019, se registró una densidad promedio de individuos de $607.40 \pm 99.88 \mathrm{ind} /$ ha (promedio \pm error estándar) dentro de los 18 transectos realizados. El año 2019 presentó en promedio una mayor densidad (750 \pm 413.22 $\mathrm{ind} / \mathrm{ha})$ que el año 2018 (493.33 $\pm 167.54 \mathrm{ind} /$ ha). La abundancia y densidades de asteroideos por transecto se muestran en la Tabla 1.

Del total de individuos de $A$. planci observados incluyendo los buceos errantes y los transectos $(\mathrm{N}=129)$, el $63 \%(\mathrm{~N}=$ 83) se encontraba alimentándose de diferentes

TABLA 1

Abundancia y densidad de Acanthaster planci registrada en los transectos realizados en El Corralito (cada uno de $75 \mathrm{~m}^{2}$ )

TABLE 1

Abundance and density of Acanthaster planci recorded along transects ( $75 \mathrm{~m}^{2}$ each) in El Corralito

\begin{tabular}{|c|c|c|c|}
\hline \multicolumn{2}{|c|}{ Fecha de la evaluación (año/mes) } & \multirow{2}{*}{$\begin{array}{c}\text { Abundancia de individuos (No.) } \\
1\end{array}$} & \multirow{2}{*}{$\begin{array}{c}\text { Densidad (ind/ha) } \\
133.33\end{array}$} \\
\hline 2018 & Agosto & & \\
\hline & & 0 & 0 \\
\hline & & 0 & 0 \\
\hline & & 0 & 0 \\
\hline & & 12 & 1600 \\
\hline & & 2 & 266.67 \\
\hline & Septiembre & 6 & 800 \\
\hline & & 3 & 400 \\
\hline & & 6 & 800 \\
\hline & & 7 & 933.33 \\
\hline \multirow[t]{9}{*}{2019} & Mayo & 27 & 3600 \\
\hline & Septiembre & 4 & 533.33 \\
\hline & & 1 & 133.33 \\
\hline & & 4 & 533.33 \\
\hline & & 0 & 0 \\
\hline & Octubre & 4 & 533.33 \\
\hline & & 2 & 266.67 \\
\hline & & 3 & 400 \\
\hline & Total & 82 & $607.40 \pm 853.40($ promedio \pm E.E. $)$ \\
\hline
\end{tabular}




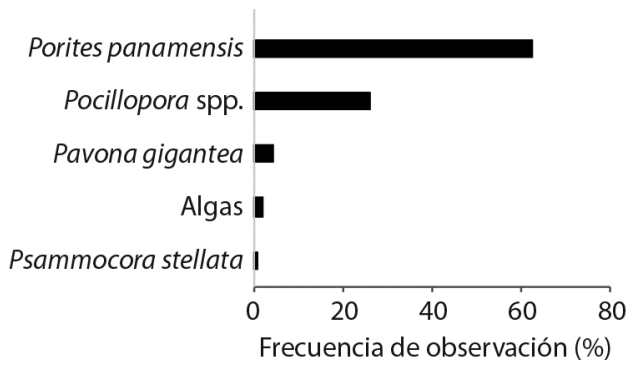

Fig. 2. Componentes de la dieta de Acanthaster planci identificados durante censos visuales en El Corralito entre 2017 y 2019.

Fig. 2. Diet components of Acanthaster planci identified during visual censuses in El Corralito between 2017 and 2019.

componentes: colonias de $P$. panamensis $(\mathrm{N}=$ $52)$, Pocillopora spp. $(\mathrm{N}=22)$, P. gigantea $(\mathrm{N}$ $=4)$, algas $(\mathrm{N}=2)$ y Psammocora stellata $(\mathrm{N}$ =1) (Fig. 2). Solamente en una ocasión no fue posible confirmar si el animal estaba o no alimentándose por la ubicación de éste dentro de un escondite, lo que dificultó la categorización. Los asteroideos restantes que no se estaban alimentando, se asociaron a más de siete sustratos diferentes, con el mayor porcentaje de ellos observados sobre roca $(26 \%, \mathrm{~N}=12)$, seguido por bloque (15\%, N =7), coral vivo $(15 \%, \mathrm{~N}=7)$, alga calcárea $(15 \%, \mathrm{~N}=7)$, arena $(8 \%, \mathrm{~N}=4)$, coral muerto $(8 \%, \mathrm{~N}=4)$ y otros sustratos (pedacería de coral y conchas, $13 \%, \mathrm{~N}=6$ ).

A lo largo del tiempo de estudio se observó una variación en la alimentación coralívora de A. planci (Fig. 3). En 2017, la mayoría de las observaciones de consumo ( $53 \%, \mathrm{~N}=26$ observaciones de alimentación activa) se presentaron sobre las especies del género Pocillopora y en menor proporción sobre $P$. panamensis (47 $\%)$. En este año, no se observaron asteroideos alimentándose activamente de colonias de $P$. gigantea, aunque ya había evidencia (basada en la ocurrencia de lesiones) del consumo sobre ellas. En 2018 se observó un aumento hasta 60 $\%$ en la depredación sobre $P$. panamensis $(\mathrm{N}=$ 20 observaciones de alimentación activa) y 80 $\%$ para $2019(\mathrm{~N}=35)$. Finalmente, el $15 \%$ de los individuos registrados en 2018, se alimentaron de colonias de $P$. gigantea, mientras que, en el 2019 , el $3 \%$ de los individuos consumieron este recurso. En el caso de Pocillopora spp. se observó una disminución en el consumo en el tiempo, de $53 \%$ en 2017 a $11 \%$ en 2019.

Respecto a la prevalencia de la depredación dentro de la comunidad coralina de El Corralito a lo largo del estudio se diagnosticaron 827 colonias de Pocillopora spp., P. panamensis, $P$. gigantea y $P$. stellata, de las cuales

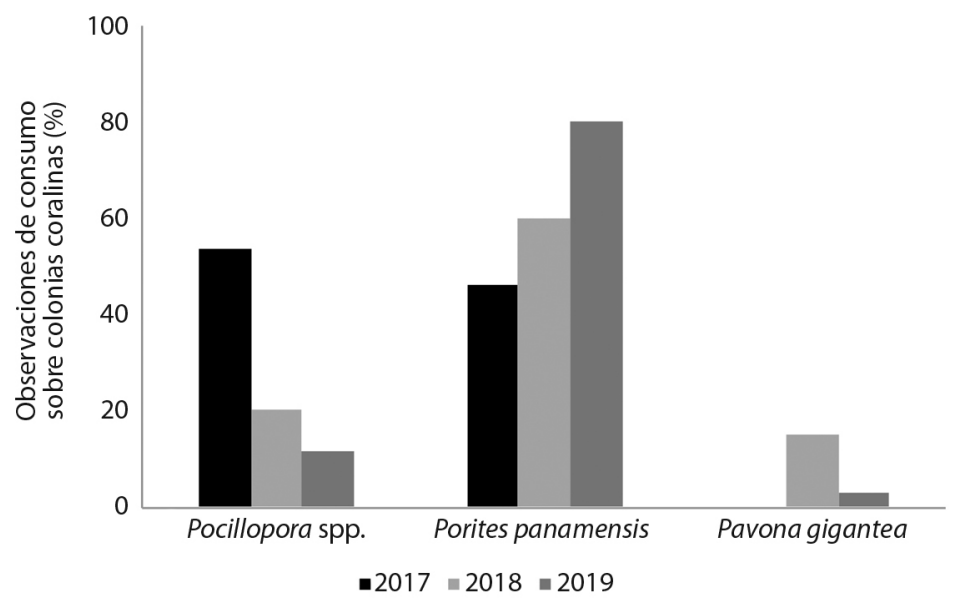

Fig. 3. Observaciones temporales en el consumo de corales durante los eventos de alimentación de Acanthaster planci en El Corralito entre los años 2017 y 2019.

Fig. 3. Temporal observations in coral consumption during feeding events by Acanthaster planci in El Corralito during the years 2017 and 2019 . 
490 presentaban la lesión característica de la depredación por $A$. planci, equivalente a una prevalencia de $59 \%$. Las diferencias en la prevalencia en el tiempo fueron estadísticamente significativas $\left(\mathrm{U}_{(10,8)}=17, \mathrm{P}=0.04\right)$, con una mediana en el año 2018 menor $(29.27 \%, \mathrm{~N}=$ 10 censos) que la reportada en $2019(74.28 \%$, $\mathrm{N}=$ ocho censos).

En la comunidad de Pocillopora spp. (N $=448$ colonias), los valores fueron significativamente mayores en $2019\left(\mathrm{U}_{(8,7)}=10.5, \mathrm{P}=\right.$ 0.04 ) cuando se observó una mediana de 74.14 $\%(\mathrm{~N}=223)$, mientras que en el año 2018 la mediana de esta comunidad depredada por el asteroideo fue de $51.85 \%(\mathrm{~N}=225)$. Si bien, entre 2018-2019, el incremento en la prevalencia de la depredación fue evidente en las especies de corales, no fue significativo para $P$. panamensis, ni P. gigantea (Fig. 4). En el caso de $P$. gigantea, la mediana de la prevalencia poblacional fue $100 \%(\mathrm{~N}=24$ colonias $)$ en 2018 y 2019. En la población de P. panamensis, la mayoría de las colonias presentaron señales de depredación en los dos años, observándose en 2019 el valor más alto de la mediana poblacional $(78.80 \%)$ respecto al $2018(42.74 \%)$. Durante el estudio solamente se registró una colonia de $P$. stellata dentro de los transectos y esta tenía señales de depredación por el asteroideo en 2019.

A lo largo del estudio, de las 490 colonias coralinas depredadas por A. planci, 304 (62\%) presentaron lesiones de pérdida de tejido subagudas, lo cual sugiere un tiempo de evolución de semanas, mientras que 151 colonias (30.8 $\%)$ presentaron una condición aguda, indicativo de que la depredación habría ocurrido horas atrás de las observaciones. En las colonias restantes $(\mathrm{N}=35,7.20 \%)$ se registraron lesiones de pérdida de tejido agudas y subagudas, que indican depredación activa y depredación pasada sobre las colonias. Se resalta que en 2019 el porcentaje de lesiones subagudas incrementó desde $55.96 \%$ hasta $66.56 \%$, con una disminución de las lesiones agudas de 34.92 hasta $27.75 \%$ (Fig. 5).

Finalmente, el seguimiento de la depredación de colonias de $P$. gigantea se realizó de manera parcial en siete de ellas puesto que el inicio del estudio fue posterior al comienzo de la depredación por parte de los asteroideos. La información del tamaño de las colonias y el porcentaje de tejido vivo observado 81 días después de iniciado el seguimiento se muestra en la Tabla 2. Solamente una de las colonias (colonia 5, diámetro mayor $=4.9 \mathrm{~m}$, Tabla 2 ) se encontraba intacta al inicio de la observación (septiembre de 2018) y sin individuos de A. planci en su cercanía, por lo que se pudo registrar el proceso completo de colonización y muerte causada por el asteroideo.

En la Fig. 6 se muestra esta secuencia la cual inicia con la colonia en buen estado de salud en noviembre de 2018 (Fig. 6A), una depredación inicial el 21 febrero de 2019 cuando pocos individuos (menos de cinco) depredan la base de la colonia (Fig. 6B), y finalmente el 13 de mayo de 2019 se observó

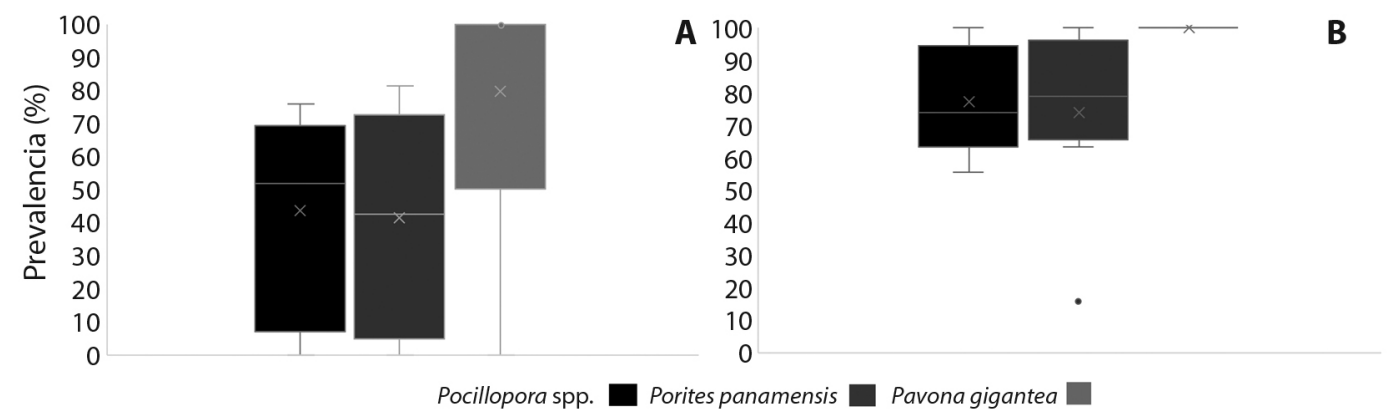

Fig. 4. Prevalencia de colonias de coral depredadas por Acanthaster planci en El Corralito entre los años A. 2018 y B. 2019.

Fig. 4. Prevalence of predated coral colonies by Acanthaster planci in El Corralito in A. 2018 and B. 2019. 
TABLA 2

Colonias de Pavona gigantea monitoreadas en El Coralito entre 2018 y 2019

TABLE 2

Monitored Pavona gigantea colonies in El Corralito between 2018 and 2019

\begin{tabular}{ccccc} 
Colonia & $\begin{array}{c}\text { Diámetro mayor } \\
(\mathrm{m})\end{array}$ & $\begin{array}{c}\text { Diámetro menor } \\
(\mathrm{m})\end{array}$ & $\begin{array}{c}\text { Porcentaje de coral vivo } \\
\text { final en } 2019(\%)\end{array}$ & $\begin{array}{c}\text { Área de la colonia } \\
\left(\mathrm{m}^{2}\right)\end{array}$ \\
1 & 2.80 & 3.20 & 1 & 14.07 \\
2 & 1.80 & 2.50 & 1 & 7.07 \\
3 & 3.41 & 2.42 & 1 & 12.96 \\
4 & 2.28 & 3.49 & 1 & 12.49 \\
5 & 4.90 & 3.35 & 1 & 25.78 \\
6 & 2.85 & 2.73 & 2 & 12.22 \\
7 & 1.62 & 1.72 & 1 & 4.37 \\
8 & 2.35 & 1.58 & 10 & 5.83 \\
\hline
\end{tabular}

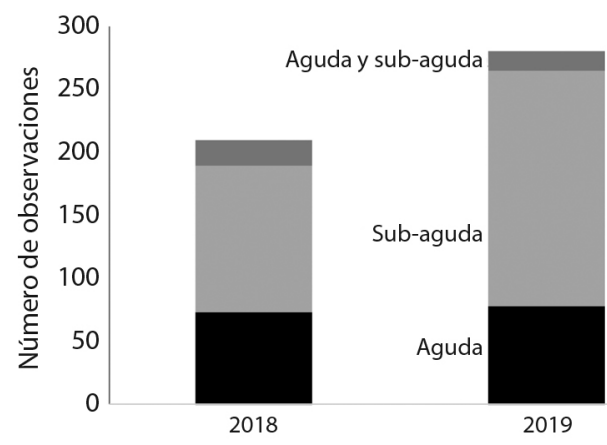

Fig. 5. Tipos de lesiones causadas por Acanthaster planci sobre colonias coralinas en El Corralito en 2018 y 2019.

Fig. 5. Injuries inflicted by Acanthaster planci on coral colonies in El Corralito in 2018 and 2019.

su depredación completa (Fig. 6C). Lo anterior indica que el proceso de consumo de la colonia (área estimada de $25 \mathrm{~m}^{2}$ ), ocurrió en 81 días, a una tasa de $0.31 \mathrm{~m}^{2} /$ día. Para el mes de mayo de 2019, las ocho colonias monitoreadas presentaban una mortalidad estimada de más del 75 \% de su área. En la Fig. 6 (D-F) se observan tres colonias depredadas por varios individuos de A. planci.

\section{DISCUSIÓN}

El incremento en la abundancia de $A$. planci y la mortalidad que genera su depredación sobre las colonias coralinas es una de las amenazas que actualmente presentan algunos arrecifes del océano Pacífico (Leray, Béraud, Anker, Chancerelle, \& Mills, 2012; Baird, Pratchett, Hoey, Herdiana, \& Campbell, 2013). En el presente trabajo se describe a lo largo de tres años, el primer evento de depredación masiva asociado al incremento en el tamaño poblacional de este asteroideo ocurrido en un arrecife del Archipiélago Espíritu Santo, México.

En El Corralito, la densidad promedio de A. planci $(607.40 \pm 99.88 \mathrm{ind} / \mathrm{ha})$ registrada en este trabajo durante los años 2018 y 2019 , se encuentra por debajo del valor reportado para este mismo lugar de $1500 \mathrm{ind} / \mathrm{ha}$ en el año 2017 estimado a través de un buceo errante (Rodríguez-Villalobos \& Ayala-Bocos, 2018). El incremento en la densidad del asteroideo y la depredación sobre las colonias coralinas en el arrecife El Corralito es evidente en comparación con los primeros registros de la población por Dana y Wolfson (1970), quienes observaron valores entre 70 y $160 \mathrm{ind} / \mathrm{ha}$ en sitios localizados al sur del arrecife de estudio. Este incremento en la densidad de A. planci ya había sido advertido para el sur del golfo de California por Hérrero-Pérezrul (2008) quien reportó los valores más altos de densidad (220 $\pm 16 \mathrm{ind} / \mathrm{ha}$ ) hasta el año 2008. En la actualidad, la densidad de este asteroideo supera los valores registrados para la bahía de La Paz en 2005 de $15.4 \pm 10.6 \mathrm{ind} / \mathrm{ha}$ (Reyes-Bonilla, González-Azcárraga, \& Rojas-Sierra, 2005), así como el promedio histórico reportado para 


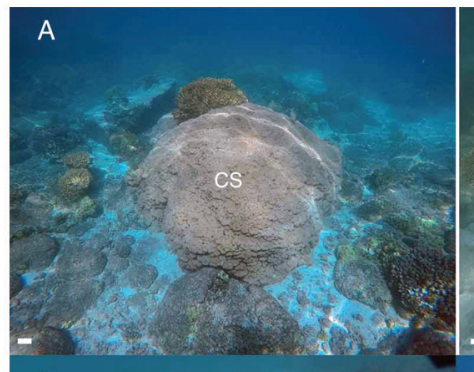

$\mathrm{D}$

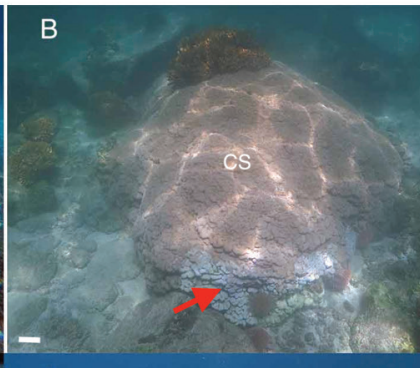

E

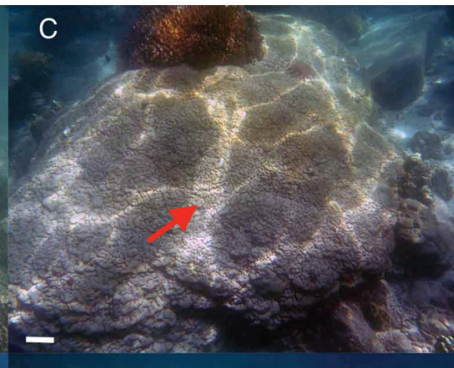

F
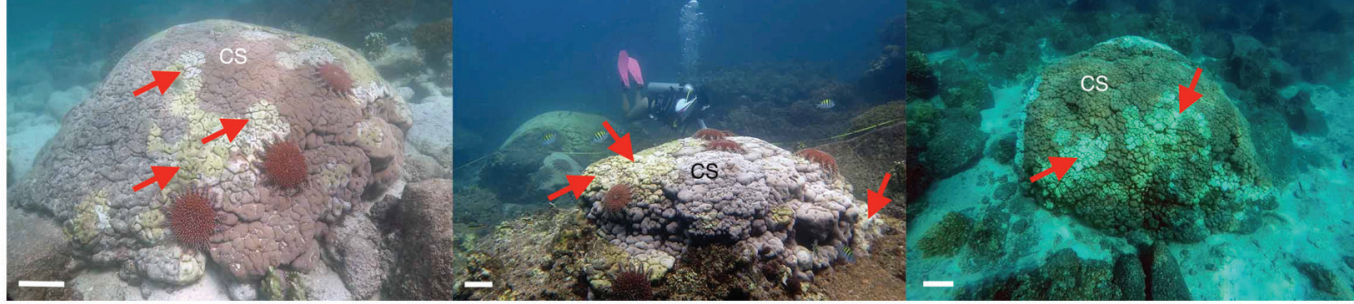

Fig. 6. Evolución de la depredación (flechas rojas indican el esqueleto expuesto de coral) de colonias de Pavona gigantea por Acanthaster planci en El Corralito. A. 3 de noviembre de 2018. B. 21 de febrero de 2019. C. 13 de mayo de 2019, no hay tejido vivo en la colonia. D-F. Colonias de Pavona gigantea depredadas por numerosos individuos de la estrella corona de espinas. Escala: $15 \mathrm{~cm}$. CS: coral sano.

Fig. 6. Evolution of the predation (red arrows indicate the exposed skeleton of the coral) on Pavona gigantea colonies by Acanthaster planci in El Corralito. A. November 3, 2018. B. February 21, 2019. C. May 13, 2019, no coral tissue's remnants. D-F. Pavona gigantea colonies predated by Crown of thorns individuals. Scale bar: $15 \mathrm{~cm}$. CS: healthy coral.

El Corralito entre 2005 y 2016, que de acuerdo con Rodríguez-Villalobos, Hernández-Carreón, Rojas-Montiel, Reyes-Bonilla y Weaver (en prensa) es de $18.94 \pm 8.06 \mathrm{ind} / \mathrm{ha}$.

La densidad de $A$. planci registrada hasta 2019 en El Corralito dentro del Archipiélago Espíritu Santo es varias veces superior a otros lugares del golfo de California. Por ejemplo, en la zona de Santa Rosalía, Reyes-Bonilla et al. (2005) registraron un promedio de $60 \pm 29 \mathrm{ind} /$ ha, mientras que en Loreto se observaron $75 \pm$ $36 \mathrm{ind} / \mathrm{ha}$ en 2004. Por su parte, Luna-Salguero y Reyes-Bonilla (2010) documentaron un promedio de $102 \pm 24 \mathrm{ind} / \mathrm{ha}$ en Loreto y $44 \pm$ $18 \mathrm{ind} /$ ha en Ligüi durante 2007. La situación actual de $A$. planci en El Corralito podría ser indicativo de un incremento poblacional excesivo para el equilibrio del ecosistema, dado que supera los niveles establecidos por Moran y De'ath (1992) para la Gran Barrera Arrecifal de Australia y por Glynn (1973) para el Pacífico de Panamá según los cuales, el crecimiento de la población del asteroideo excede las tasas de crecimiento y recuperación de los arrecifes.

En el caso de El Corralito, además de las altas densidades registradas en este estudio durante tres años consecutivos (2017-2019), el daño a los corales y el deterioro del arrecife ha sido evidente con mortalidades de colonias asociadas con la depredación de $A$. planci mayores al $50 \%$ y con lesiones agudas, que indican una depredación activa en el lugar. Esto es una aproximación al daño general sobre el arrecife, considerando la función ecológica que desempeñan los corales vivos en el ecosistema y la relación que presentan con numerosas especies que dependen del tejido y el mucus de las especies (Rice et al., 2019). En el POT y en el golfo de California, no existían reportes de depredación masiva de corales asociados con incrementos en la densidad de este asteroideo que hayan ocasionado pérdidas representativas de coral vivo en los arrecifes, aunque las señales de la depredación se han observado 
en arrecifes de Panamá, Costa Rica y México, con un registro no sistemático de ellas (Dana \& Wolfson, 1970; Glynn 1990). De acuerdo con DeVantier y Done (2007), en la Gran Barrera Arrecifal, poblaciones pequeñas de la estrella corona de espinas (por debajo de 1 ind/ ha) depredan selectivamente sobre colonias ramificadas y de rápido crecimiento, por lo que las señales de la coralivoría se desvanecen debido al crecimiento de las colonias, dejando pocos efectos ecológicos sobre el ecosistema. Las densidades por debajo de niveles dañinos para el arrecife reportadas con anterioridad en el golfo de California (Reyes-Bonilla et al., 2005; Luna-Salguero \& Reyes Bonilla, 2010), podrían explicar la prácticamente nula evidencia anterior de daño causado por la estrella corona de espinas en el Pacífico mexicano, en donde pocos registros de depredación apenas son registrados en la literatura (Dana \& Wolfson, 1970) y una sola estimación da cuenta del porcentaje $(<1 \%)$ de lesiones de pérdida de tejido asociada con este depredador (Rodríguez-Villalobos et al., 2015) sobre colonias del género más abundante y de rápido crecimiento del POT, Pocillopora (Reyes-Bonilla, 2003). El incremento de las observaciones de mortalidad coralina a partir de 2017 reportadas en este trabajo podría explicarse por el incremento de la población de $A$. planci, pues cuando las poblaciones crecen exponencialmente por encima de cientos o miles de individuos por hectárea, los asteroideos comienzan a depredar de manera general sobre todas las colonias de coral, incluyendo aquellas con crecimiento masivo (DeVantier \& Done, 2007), dejando amplias regiones de pérdida de tejido y sobrecrecimiento algal, que permiten hacer reconstrucciones de los eventos de depredación por medio de la observación y cuantificación de las lesiones sobre las colonias coralinas.

En El Corralito la depredación de $A$. planci sobre los corales ha presentado un patrón de preferencia diferencial en el tiempo. Los primeros registros observacionales que se tuvieron en 2017 se asociaron con la depredación sobre las colonias de mayor tamaño en el arrecife, es decir las de $P$. gigantea, que al poseer las menores coberturas coralinas en el sitio $(<1 \%$, obs. pers.), por consecuencia sufrieron las mayores reducciones, alcanzando una mortalidad promedio cercana al $80 \%$ en el año 2018, y del $100 \%$ en 2019 registrada en este trabajo. Pavona gigantea posee un crecimiento lento (con tasas menores de $1 \mathrm{~cm} /$ año), ofrece un tejido denso y una mayor accesibilidad para $A$. planci, ya que son colonias de crecimiento masivo y planas en comparación con las colonias ramificadas de Pocillopora spp. Adicionalmente, $P$. gigantea no presenta organismos simbiontes como los cangrejos del género Trapezia que incrementan la defensa contra depredadores en las colonias ramificadas (Keesing, 1990). La depredación del asteroideo sobre colonias del género Pavona ha sido reportada previamente para arrecifes del POT por Maté (2003), quien además señala su predilección sobre especies de Porites e incluso Pocillopora. De acuerdo con el seguimiento de la prevalencia de lesiones asociadas con la depredación, en El Corralito el segundo coral más consumido (70 \% mediana de prevalencia de individuos afectados) fue Pocillopora spp., el cual es el género principal de construcción arrecifal en el POT (Reyes-Bonilla, 2003). Este género de coral posee forma ramificada y muestra un rápido crecimiento (por encima de $10 \mathrm{~cm} / a n ̃ o)$, que si bien se ha registrado como parte de la dieta de $A$. planci en otros trabajos (Pratchett, 2001; Rodríguez-Villalobos et al., 2015), no siempre es la elección principal debido a la asociación que presenta con crustáceos simbiontes como cangrejos y peces damisela (Maté, 2003), que defienden la colonia de la acción de depredadores (Pratchett, 2001). En el Pacífico americano, pese a la dominancia en la cobertura de especies del género Pocillopora, si bien no se ha presentado una alta mortalidad asociada a $A$. planci, estos si son consumidos por el equinodermo (Glynn, 1983, 1990). Los eventos de depredación se han asociado principalmente con eventos naturales tales como El Niño o huracanes, que ocasionan una remoción del grueso marco arrecifal conformado por las colonias de Pocillopora, permitiendo el acceso 
de los asteroideos a la parte central más interna de los arrecifes (Glynn, 1983).

En El Corralito, a partir del año 2018 se observó un incremento en la depredación de $P$. panamensis, un género que ha sido reportado como uno de los menos preferidos por A. planci (Keesing, 1990; De'ath \& Moran, 1998) en arrecifes del Pacífico. Diversos factores se han relacionado con las preferencias de consumo de Acanthaster, tales como el contenido nutricional de los corales, la forma de crecimiento colonial, las defensas del coral (como filamentos mesentéricos o nematocistos), pero también otros factores ecológicos (e.g. asociación con otras especies, disponibilidad/rareza del recurso) pueden determinar elecciones particulares en su alimentación (Keesing, 1990; Pratchett, 2007). En El Corralito, probablemente en relación a la alta densidad de individuos de $A$. planci alimentándose como efecto de competencia intraespecífica, es probable que los asteroideos presenten cambios en sus dietas haciendo que el consumo sobre colonias de especies menos preferidas incremente (Pratchett, 2007). De acuerdo con el aumento en la prevalencia de las lesiones en Pocillopora y Pavona (lo que sugiere disminución en su disponibilidad), los asteroideos incrementaron el consumo de las colonias más pequeñas y distribuidas de manera dispersa en el arrecife, como $P$. panamensis. Esta observación tiene una relevancia ecológica para la conservación de los arrecifes del sur del golfo de California, pues demuestra que $A$. planci es un consumidor generalista, lo que puede ocasionar pérdidas masivas de cobertura de coral en los arrecifes de tamaño pequeño y distribuidos de manera dispersa como los que se presentan en la región (Reyes-Bonilla, 2003).

El seguimiento de la depredación de la colonia de $P$. gigantea y el consumo del tejido a razón de $0.31 \mathrm{~m}^{2} /$ día, debe ponerse en perspectiva con relación a la densidad observada en El Corralito para la estimación del daño potencial que genera $A$. planci sobre el arrecife. De acuerdo con Reyes-Bonilla y Calderon-Aguilera (1999), individuos de tallas promedio cercanas a los $14 \mathrm{~cm}$ de diámetro de disco (16 $\mathrm{cm}$ es el promedio de talla registrada en El Corralito, datos no mostrados), pueden consumir de manera individual hasta $4.2 \mathrm{~m}^{2} \mathrm{de}$ coral/año. Considerando la densidad promedio reportada en este estudio de $607 \mathrm{ind} / \mathrm{ha}$, la pérdida de coral sería de más de 0.26 ha al año, lo cual equivale al área total de coral estimada con relación al $20 \%$ de cobertura, en un arrecife de 1.3 ha totales. Es importante mencionar que la pérdida masiva de cobertura de coral vivo en los arrecifes tras eventos de depredación u otro tipo de disturbio natural, la recuperación de las especies coralinas es lenta y dependiendo de las especies de coral y la extensión del arrecife afectadas, podría tomar entre un par y más de 100 años (Pratchett et al., 2017). Hasta septiembre de 2020 no se observaron señales de recuperación en las colonias de coral muertas y el evento de depredación masiva continuaba extendiéndose hacia sectores dominados por Pocillopora spp. previamente no depredados (obs. pers.). La recuperación del tejido coralino en especies de Pocillopora spp. es posible bajo condiciones óptimas para los individuos (Rodríguez-Villalobos, Work, \& CalderonAguilera, 2016), lo cual requiere el control de competidores coralinos como las algas, por lo que se requiere evaluar las poblaciones de herbívoros presentes en el arrecife y monitorear el estado de recuperación de las colonias y las comunidades de otras especies arrecifales en el tiempo.

Si como se ha observado en algunos brotes poblacionales de Acanthaster spp. ocurridos en la Gran Barrera Arrecifal de Australia posterior a estos eventos, ocurren disminuciones drásticas en el número de individuos del asteroideo (Pratchett, 2005), se podría esperar que en los próximos años se observen en El Corralito densidades inferiores a las reportadas desde 2017 y que tras de esto la recuperación del arrecife pueda alcanzarse. Dada la cercanía que presentan las comunidades coralinas y los arrecifes dentro del Archipiélago Espíritu Santo, se debe considerar también la posibilidad de que los individuos se trasladen a arrecifes cercanos que ofrezcan facilidades en el acceso de presa para los asteroideos y de esta manera se 
extienda el evento de depredación masiva. Sin embargo, a lo largo del estudio de los brotes de Acanthaster spp. se han observado diferencias en la ocurrencia y severidad de estos eventos entre arrecifes de la Gran Barrera Arrecifal de Australia, sin que se tenga certeza clara sobre las razones por las que se originan crecimientos de la población de manera específica sobre un arrecife en particular (Pratchett et al., 2017). Sin el entendimiento del origen del crecimiento poblacional, no es apropiado predecir el comportamiento de los individuos de $A$. planci en el golfo de California.

En conclusión, la alta densidad de individuos de $A$. planci, la presencia de lesiones agudas sobre las colonias, el número de individuos activamente alimentándose de corales, así como las altas prevalencias de depredación presentes en las colonias coralinas en el arrecife, indican que actualmente El Corralito experimenta un brote poblacional de la estrella corona de espinas que es perjudicial para la permanencia del ecosistema. Lo anterior amerita que exista un monitoreo continuo y un estudio estricto de la población de asteroideos en el sur del golfo de California, cuya información sea utilizada para la formulación de proyectos para mitigar el deterioro y la potencial extensión del evento a otros arrecifes. De igual manera, se requiere que los pobladores locales comprendan la problemática sin realizar intervenciones perjudiciales sobre estos asteroideos y participen activamente en la vigilancia de los arrecifes en detección de amenazas sobre ellos.

Declaración de ética: los autores declaran que todos están de acuerdo con esta publicación y que han hecho aportes que justifican su autoría; que no hay conflicto de interés de ningún tipo; y que han cumplido con todos los requisitos y procedimientos éticos y legales pertinentes. Todas las fuentes de financiamiento se detallan plena y claramente en la sección de agradecimientos. El respectivo documento legal firmado se encuentra en los archivos de la revista.

\section{AGRADECIMIENTOS}

A las autoridades y el personal del Parque Nacional Zona Marina Archipiélago Espíritu Santo, por las facilidades logísticas. Miembros del staff y estudiantes asociados a la Sociedad de Historia Natural Niparajá, A.C., y a ECO: ecosistemas y conservación (Proazul terrestre A.C.) quienes apoyaron en las labores de campo.

\section{RESUMEN}

\author{
Depredación masiva por Acanthaster planci \\ en el arrecife El Corralito, golfo de California: \\ amenaza a corto plazo
}

Introducción: una de las amenazas actuales para los arrecifes de coral es la pérdida de cobertura de coral vivo. La depredación masiva asociada con los brotes poblaciones de la estrella corona de espinas, amenaza la permanencia de los arrecifes. Desde 2017, ha habido evidencia de un incremento en la densidad de el asteroideo y de un incremento en la mortalidad coralina en el sur del golfo de California. Objetivo: describir el primer evento de depredación sobre corales por Acanthaster planci en la isla Espíritu Santo, golfo de California, México. Métodos: visitamos el arrecife El Corralito nueve veces entre 2017 y 2019, realizando censos visuales en transectos errantes y de banda $(25 \times 3 \mathrm{~m})$ para determinar la densidad de la estrella, su comportamiento y daño. Monitoreamos la depredación sobre las colonias de Pavona gigantea durante siete meses. Resultados: la densidad promedio de individuos en El Corralito en 2018-19 fue 607.40 ind/ha. La depredación del asteoroideo fue evidente en más del $60 \%$ de los individuos diagnosticados $(\mathrm{N}=827)$, con las afectaciones más grandes en las colonias de $P$. gigantea (80\%). El 63\% de los 129 de los individuos del asteroideo observados estaban alimentándose principalmente sobre Porites panamensis $(68 \%$ de los casos). Las lesiones agudas indican que el evento de depredación está activo. En 81 días, se perdieron $25 \mathrm{~m}^{2}$ de una de las colonias monitoreadas. Conclusiones: Existe un evento de brote poblacional activo con consecuencias negativas significativas sobre el arrecife El Corralito, el cual puede ocasionar pérdida de cobertura de coral en pocos meses. El monitoreo y el manejo son necesarios para establecer las razones que llevaron a brote poblacional y para controlarlo. 
Palabras clave: mortalidad; coralivoría; degradación; brote poblacional; lesiones.

\section{REFERENCIAS}

Baird, A.H., Pratchett, M.S., Hoey, A.S., Herdiana, Y., \& Campbell, S.J. (2013). Acanthaster planci is a major cause of coral mortality in Indonesia. Coral Reefs, $32(3), 803-812$.

Birkeland, C., \& Lucas, J.S. (1990). Acanthaster planci: Major management problem ofcoral reefs. Boston, Estados Unidos: CRC Press

Birkeland, C. (2019). Chapter 2. Global status of coral reefs: In combination, disturbances and stressors become ratchets. En C. Sheppard (Ed.), World seas: An environmental evaluation. Volume III: Ecological issues and environmental impacts (pp. 35-56). Reino Unido: Elsevier.

Branham, J.M., Reed, S.A., \& Bailey, J.H. (1971). Coraleating sea stars Acanthaster planci in Hawaii. Science, $172,1155-1157$.

Bruno, J.F., \& Selig, E.R. (2007). Regional decline of coral cover in the Indo-Pacific: Timing, extent, and subregional comparisons. PloS ONE, 8, e711.

Chesher, R.H. (1969). Destruction of Pacific corals by the sea star Acanthaster planci. Science, 165(3890), 280-283.

CONANP. (2014). Programa de Manejo Parque Nacional exclusivamente la zona marina del Archipiélago de Espiritu Santo. México: SEMARNAT, CONANP.

Dana, T., \& Wolfson, A. (1970). Eastern Pacific Crownof-thorns starfish populations in the lower Gulf of California. Transactions of the San Diego Society of Natural History, 16(4), 83-90.

De'ath, G., \& Moran, P.J. (1998). Factors affecting the behaviour of crown-of-thorns Starfish (Acanthaster planci L.) on the Great Barrier Reef: 2: Feeding preferences. Journal of Experimental Marine Biology and Ecology, 220, 107-126.

De'ath, G., Fabricius, K.E., Sweatman, H., \& Puotinen, M. (2012). The 27-year decline of coral cover on the Great Barrier Reef and its causes. Proceedings of the National Academy of Sciences, 109(44), 17995-17999.

DeVantier, L.M., \& Done, T.J. (2007). Inferring past outbreaks of the crown-of-thorns seastar from scar patterns on coral heads. En R.B. Aronson (Ed.), Geological approaches to coral reef ecology (pp. 85-125). Nueva York: Springer.

Endean, R. (1969). Report on investigations made into aspects of the current Acanthaster planci (crown-of-thorns) infestations of certain reefs of the Great Barrier Reef. Fisheries Branch.

Endean, R. (1974). Acanthaster planci on the Great Barrier Reef. Proceedings of the Second International Coral Reef Symposium, 1, 563-576.

Enochs, I.C., \& Glynn, P.W. (2017). Corallivory in the eastern Pacific. En P. Glynn P., D. Manzello, \& I. Enochs (Eds.), Coral Reefs of the Eastern Tropical Pacific (pp. 315-337). Dordrecht: Springer.

França, F.M., Benkwitt, C.E., Peralta, G., Robinson, J.P.W., Graham, N.A.J., Tylianakis, J.M., ... Barlow, J. (2020). Climatic and local stressor interactions threaten tropical forests and coral reefs. Philosophical Transactions of the Royal Society B, 375(1794), 20190116.

Glynn, P.W. (1973). Acanthaster: Effect on coral reef growth in Panama. Science, 180(4085), 504-506.

Glynn, P.W. (1976). Some physical and biological determinants of coral community structure in the eastern Pacific. Ecology Monographs, 46, 431-456.

Glynn, P.W. (1983). Extensive "bleaching" and death of reef corals on the Pacific coast of Panamá. Environmental Conservation, 10(2), 149-154.

Glynn, P.W. (1990). Coral mortality and disturbances to coral reefs in the tropical eastern Pacific. Elsevier Oceanography Series, 52, 55-126.

Goldberg, J., \& Wilkinson, C. (2004). Global threats to coral reefs: coral bleaching, global climate change, disease, predator plagues and invasive species. En C. Wilkinson (Ed.), Status of coral reefs of the world, 2004 (pp. 67-92). Townsville: Australian Institute of Marine Science.

Haszprunar, G., \& Spies, M. (2014). An integrative approach to the taxonomy of the crown-of-thorns starfish species group (Asteroidea: Acanthaster): A review of names and comparison to recent molecular data. Zootaxa, 3841(2), 271-284.

Haszprunar, G., Vogler, C., \& Wörheide, G. (2017). Persistent gaps of knowledge for naming and distinguishing multiple species of crown-of-thorns-seastar in the Acanthaster planci species complex. Diversity, $9(22), 1-10$.

Henry, L.A., \& Hart, M. (2005). Regeneration from injury and resource allocation in sponges and corals -a review. International Review of Hydrobiology, 90, 125-158.

Herrero-Pérezrul, M.D. (2008). Diversity and abundance of reef macro invertebrates (Mollusca; Echinodermata) in the southern Gulf of California, México. Proceedings of the 11th International Coral Reef Symposium, 26, 7-11. 
Hoey, A.S., Howells, E., Johansen, J.L., Hobbs, J.P.A., Messmer, V., McCowan, D.M., Wilson, S.K., \& Pratchett, M.S. (2016). Recent advances in understanding the effects of climate change on coral reefs. Diversity, $8(2), 12$.

Horton, T., Kroh, A., Ahyong, S., Bailly, N., Boyko, C.B., Brandão, S.N., ... Zhao, Z. (2020). World Register of Marine Species (WoRMS). WoRMS Editorial Board. Recuperado de https://www.marinespecies.org

Kayal, M., Vercelloni, J., Lison De Loma, T., Bosserelle, P., Chancerelle, Y., Geoffroy, S., ... \& Adjeroud, M. (2012). Predator crown-of-thorns starfish (Acanthaster planci) outbreak, mass mortality of corals, and cascading effects on reef fish and benthic communities. PloS ONE, 7(10), e47363.

Keesing, J.K. (1990). Feeding biology of the crown-ofthorns starfish, Acanthaster planci (Linnaeus) (Doctoral dissertation). James Cook University, Australia.

Leray M., Béraud, M., Anker, A., Chancerelle, Y., \& Mills, S.C. (2012). Acanthaster planci outbreak: Decline in coral health, coral size structure modification and consequences for obligate decapod assemblages. PLoS ONE, 7(4), 1-10.

Luna-Salguero, B.M., \& Reyes-Bonilla, H. (2010). Estructura comunitaria y trófica de las estrellas de mar (Echinodermata: Asteroidea) en arrecifes rocosos de Loreto, Golfo de California, México. Hidrobiológica, 20(2), 127-134.

Maté, J.L. (2003). Corals and coral reefs of the Pacific coast of Panamá. En J. Cortés (Ed.), Latin American coral reefs (pp. 387-417). Reino Unido: Elsevier Science.

Moore R.J. (1990). Persistent and transient populations of the Crown-of-thorns starfish, Acanthaster planci. En R. Bradbury (Ed.), Acanthaster and the coral reef: A theoretical perspective. Lecture Notes in Biomathematics (Vol. 88) (pp. 236-277). Berlin, Heidelberg: Springer.

Moran, P.J. (1986). The Acanthaster phenomenon. Oceanography and Marine Biology: An Annual Review, 24, 379-480.

Moran, P.J., \& De'ath, G. (1992). Estimates of the abundance of the crown-of-thorns starfish Acanthaster planci in outbreaking and non-outbreaking populations on reefs within the Great Barrier Reef. Marine Biology, 113(3), 509-515.

Peters E.C. (2015). Diseases of coral reef organisms. En C. Birkeland (Ed.), Coral Reefs in the Anthropocene (pp. 147-178). Dordrecht: Springer.
Pratchett, M.S. (2001). Influence of coral symbionts on feeding preferences of crown-of-thorns starfish Acanthaster planci in the western Pacific. Marine Ecology Progress Series, 214, 111-119.

Pratchett, M.S. (2005). Dynamics of an outbreak population of Acanthaster planci at Lizard Island, northern Great Barrier Reef (1995-1999). Coral Reefs, 24(3), 453-462.

Pratchett, M.S. (2007) Feeding preferences of Acanthaster planci (Echinodermata: Asteroidea) under controlled conditions of food availability. Pacific Science, 61(1), 113-120.

Pratchett, M.S., Caballes, C.F., Rivera-Posada, J.A., \& Sweatman, H.P.A. (2014). Limits to understanding and managing outbreaks of crown-of-thorns starfish (Acanthaster spp.). Oceanography and Marine Biology: An Annual Review, 52, 133-200.

Pratchett, M.S., Caballes, C.F., Wilmes, J.C., Matthews, S., Mellin, C., Sweatman, H.P.A, ... Uthicke, S. (2017). Thirty years of research on crown-of-thorns starfish (1986-2016): Scientific advances and emerging opportunities. Diversity, 9(4), 41.

Randall, J.E. (1972). Chemical pollution in the sea and the crown-of-thorns starfish (Acanthaster planci). Biotropica, 4(3), 132-144.

Reyes-Bonilla, H., \& Calderon-Aguilera, L.E. (1999). Population density, distribution and consumption rates of three corallivores at Cabo Pulmo Reef, Gulf of California, Mexico. Marine Ecology, 20(3-4), 347-357.

Reyes-Bonilla, H. (2003). Coral reefs of the Pacific coast of México. En J. Cortés (Ed.), Latin American coral reefs (pp. 331-349). Amsterdam: Elsevier Science.

Reyes-Bonilla, H., González-Azcárraga, A., \& RojasSierra, A. (2005). Estructura de las asociaciones de las estrellas de mar (Asteroidea) en arrecifes rocosos del Golfo de California, México. Revista de Biología Tropical, 53(3), 233-244.

Reyes-Bonilla, H., \& López-Pérez, R.A. (2009). Corals and coral-reef communities in the Gulf of California. En M.E. Johnson \& J. Ledesma-Vásquez (Eds.), Atlas of Coastal Ecosystems in the Western Gulf of California (pp. 45-57). Tucson: University of Arizona Press.

Rice, M.M., Ezzat, L., \& Burkepile, D.E. (2019). Corallivory in the Anthropocene: Interactive effects of anthropogenic stressors and corallivory on coral reefs. Frontiers in Marine Science, 5, 525. 
Rodríguez-Villalobos, J.C., Work, T.M., Calderon-Aguilera, L.E., Reyes-Bonilla, H., \& Hernández, L. (2015). Explained and unexplained tissue loss in corals from the Tropical Eastern Pacific. Diseases of Aquatic Organisms, 116(2), 121-131.

Rodríguez-Villalobos, J.C., Work, T.M., \& Calderon-Aguilera, L.E. (2016). Wound repair in Pocillopora. Journal of Invertebrate Pathology, 139, 1-5.

Rodríguez-Vilalobos, J.C., \& Ayala-Bocos, A. (2018) Coral colonies in the eastern tropical Pacific: predation by Acanthaster cf. solaris. Pacific Conservation Biology, 24(4), 419-420.

Rodríguez-Villalobos, J.C., Hernández-Carreón, C., RojasMontiel, B., Reyes-Bonilla, H., \& Weaver, A.H. (En prensa). Temporal and spatial abundance of Acanthaster cf. solaris (Echinodermata: Asteroidea) in a national park in the Gulf of California, Mexico: 2005-2016. Pacific Science.
Rotjan, R.D., \& Lewis, S.M. (2008). Impact of coral predators on tropical reefs. Marine Ecology Progress Series, 367, 73-91.

StatSoft, Inc. (2005). STATISTICA (Data Analysis Software System), version 7.1. Recuperado de www. statsoft.com

Uthicke, S., Schaffelke, B., \& Byrne, M. (2009). A boom-bust phylum? Ecological and evolutionary consequences of density variations in echinoderms. Ecological Monographs, 79(1), 3-24.

Vogler, C., Benzie, J., Lessios, H., Barber, P., \& Wörheide, G. (2008). A threat to coral reefs multiplied? Four species of crown-of-thorns starfish. Biology Letters, 4(6), 696-699.

Work, T.M., \& Aeby, G.S. (2006). Systematically describing gross lesions in corals. Diseases of Aquatic Organisms, 70(1-2), 155-160. 